Теорія Ймовір. та Матем. Статист. Вип. 73, 2005
Theor. Probability and Math. Statist.

No. 73, 2006, Pages 109-115 S 0094-9000(07)00685-0

Article electronically published on January 17, 2007

\title{
VALUATION OF FLEXIBLE INSURANCE CONTRACTS
}

UDC 519.21

\author{
A. V. MELNIKOV, M. M. MOLIBOGA, AND V. S. SKORNYAKOVA
}

\begin{abstract}
This work is devoted to the study of insurance contracts based on risky instruments of the financial market. In the case of Black and Scholes for constant and stochastic volatility, we present specific formulas for the valuation of premiums for contracts of pure endowment with guarantee.
\end{abstract}

\section{INTRODUCTION}

This article deals with the questions of calculations involved in life insurance policies. For us, the significance lies in the innovative nature of the contracts, where insurance payments depend on the market price of some security and, consequently, can have a rather complicated risk structure. Traditionally, life insurance payments are predetermined, which simplifies the calculation of premiums on the basis of standard application of the equivalence principle (see [1, for instance). However, a new stage in the evolution of the financial system (the stage of floating exchange and interest rates) demanded insurance to be adapted to these new changeable conditions. This resulted in the creation of the already mentioned innovative schemes, called equity-linked life insurance contracts, which are valuated with the help of a risk-neutral equivalence principle (see [2]-7]). In this work, we give specific formulas for the value of policies of pure endowment with guarantee, in the case when the underlying security evolves according to a Black-Scholes model with constant and stochastic volatility. The basis for our approach comes from the methods of perfect hedging in complete markets and superhedging in incomplete ones (see [7]).

The character of flexible insurance contracts is determined by the presence of two sources of risk: mortality of the client and financial uncertainty of the securities market. Traditional insurance considers only the first source, reducing the second with the hypothesis of predetermined future payments. Vice versa, in financial economics, the first factor is ignored, and future payments in reality depend on the evolution of the market during the contract period. It follows from the above that quantitative calculations of the amounts of premiums and reserves for the innovative type of insurance must combine the methods of actuarial and financial mathematics. To this topic we devote our paper, which is a corresponding edition of the first author's presentation at the international conference commemorating the 90th birthday anniversary of B. V. Gnedenko (June 2002, Kyiv).

2000 Mathematics Subject Classification. Primary 60H30, 91B28, 91B30.

The first author was supported in part by NSERC grant G121210913. 


\section{Setup of the problem and Calculations in the Black-Scholes model}

Consider a contract of pure endowment. This contract gives the right to its holder, who is of age $x$ at the termination of the contract, to receive the amount $g$ with the condition that the holder lives to be $x+T$ old. Denote by ${ }_{T} U_{x}$ the corresponding premium, or price of the contract.

As we can see from the structure of the contract, the mortality of the client plays a key role here. The probability of survival ${ }_{t} p_{x}$ is an adequate quantitative characteristic of the mortality of the client; it is the probability that the remaining number of years to live, $T(x)$, will exceed $t$.

We will do all calculations for a pure endowment contract with the following structure of payment:

$$
g=g(S)=\max \left\{S_{T}, K\right\},
$$

where $S_{T}$ is the market value of a security at the expiration date of the contract, $T$, and $K$ is the guaranteed payment, no matter what has happened in the market during the time of the contract. All calculations below are conducted for this pure endowment contract with guarantee.

Heuristically, it is obvious that the indicated above types of randomness, or risk, have very little in common. Because of this, we will view them as independent in the probabilistic sense, which enables us to construct corresponding probability spaces for their consideration.

Assume that the "financial" probability space $(\hat{\Omega}, \hat{F}, \hat{\mathrm{P}})$ is equipped with filtration $\hat{\mathbf{F}}=\left(\hat{F}_{t}\right)_{t \geq 0}$ created by prices of our security: $\hat{F}_{t}=\sigma\left\{S_{u}, u \leq t\right\}$.

Also, we suppose that independent random variables $\left(T_{i}(x)\right)_{i \leq l_{x}}$ are given on the "insurance" probability space $(\widetilde{\Omega}, \widetilde{F}, \widetilde{P})$. These variables represent the remaining years of life of a homogeneous group of persons of age $x$. Let's define the counting mortality process:

$$
N_{t}=\sum_{i=1}^{l_{x}} I_{\left\{T_{i}(x) \leq t\right\}}
$$

and its filtration

$$
\widetilde{F}_{t}=\sigma\left\{N_{s}, s \leq t\right\}
$$

In the context of the above setup, we will consider life insurance contracts whose payments depend on the price of the security on the following probability space:

$$
(\Omega, F, \mathbf{F}, \mathrm{P})=(\hat{\Omega} \times \widetilde{\Omega}, \hat{F} \times \widetilde{F}, \hat{\mathbf{F}} \times \widetilde{\mathbf{F}}, \hat{\mathrm{P}} \times \widetilde{\mathrm{P}})
$$

In order to make any calculations, we must specify the evolution of the security $S$. First, let's look at the Black-Scholes model (the model of Geometric Brownian Motion, see [7]), supposing the interest rate $r$ to be zero for the sake of simplicity, which gives us the value of the nonrisky asset (bond) $B_{T} \equiv 1=$ const. Specifically,

$$
S_{t}=S_{0} \exp \left\{\left(\mu-\frac{\sigma^{2}}{2}\right) t+\sigma W_{t}\right\}
$$

or

$$
d S_{t}=S_{t}\left(\mu d t+\sigma d W_{t}\right),
$$

where $W=\left(W_{t}, \hat{F}_{t}\right)$ is a standard Wiener process on the space $(\hat{\Omega}, \hat{F}, \hat{\mathrm{P}}), \mu \in \mathbf{R}^{1}$ is the rate of return, and $\sigma \in \mathbf{R}_{+}$is volatility. 
Suppose an insurance company has pure endowment contracts with guarantee with a group of people with future lifetimes $T_{1}(x), \ldots, T_{l_{x}}(x)$, distributed as a random variable $T(x)$. Clearly, the cumulative payment for such a contract equals

$$
\sum_{k=1}^{l_{x}} \max \left\{S_{T}, K\right\} \cdot I_{\left\{T_{k}(x)>T\right\}}=\max \left\{S_{T}, K\right\} \cdot \sum_{k=1}^{l_{x}} I_{\left\{T_{k}(x)>T\right\}}
$$

and becomes a payment obligation on the space $(\Omega, F, \mathrm{P})$ introduced above.

Note that the corresponding contract payment for one of the persons in the group under consideration equals

$$
\frac{1}{l_{x}} \max \left\{S_{T}, K\right\} \cdot \sum_{k=1}^{l_{x}} I_{\left\{T_{k}(x)>T\right\}},
$$

and we work with the group of $l_{x}$ clients according to actuarial traditions.

According to the theory of calculations of payments in the Black-Scholes case, the derivation of the premium ${ }_{T} U_{x}$ for such a contract is reduced to averaging the corresponding contingent claim under the risk-neutral probability $\mathrm{P}^{*}$. In our case, this probability becomes $\mathrm{P}^{*}=\hat{\mathrm{P}}^{*} \times \widetilde{\mathrm{P}}$, with the density of $\hat{\mathrm{P}}^{*}$ given by

$$
\frac{d \hat{P}^{*}}{d \hat{P}}=\exp \left\{-\frac{\mu}{\sigma} W_{T}-\frac{1}{2}\left(\frac{\mu}{\sigma}\right)^{2} T\right\}
$$

which is a martingale measure for our model.

Consequently, we get

$$
\begin{aligned}
{ }_{T} U_{x} & =\frac{1}{l_{x}} \mathrm{E}^{*} \max \left\{S_{T}, K\right\} \cdot \sum_{k=1}^{l_{x}} I_{\left\{T_{k}(x)>T\right\}} \\
& =\frac{1}{l_{x}} \hat{\mathrm{E}}^{*} \max \left\{S_{T}, K\right\} \cdot \sum_{k=1}^{l_{x}} \tilde{\mathrm{E}} I_{\left\{T_{k}(x)>T\right\}} \\
& =\frac{1}{l_{x}} \hat{\mathrm{E}}^{*} \max \left\{S_{T}, K\right\} \cdot l_{x} \tilde{\mathrm{E}} I_{\{T(x)>T\}} \\
& =\frac{1}{l_{x}} \hat{\mathrm{E}}^{*} \max \left\{S_{T}, K\right\} \cdot l_{x T} p_{x} \\
& ={ }_{T} p_{x} \hat{\mathrm{E}}^{*} \max \left\{S_{T}, K\right\} .
\end{aligned}
$$

Now, considering the relation

$$
\max \left\{S_{T}, K\right\}=K+\left(S_{T}-K\right)^{+},
$$

we find that

$$
{ }_{T} U_{x}={ }_{T} p_{x} K+{ }_{T} p_{x} \hat{\mathrm{E}}^{*}\left(S_{T}-K\right)^{+} .
$$

It is well known ([7]) that $\hat{\mathrm{E}}^{*}\left(S_{T}-K\right)^{+}$is the price of a buyer's option, and this price is actually the initial capital of the minimal hedge, given by the Black-Scholes formula. Therefore,

$$
{ }_{T} U_{x}={ }_{T} p_{x} K+{ }_{T} p_{x} C_{T}(0)
$$


where

$$
\begin{gathered}
C_{T}(0)=S_{0} \Phi\left(d_{+}(0)\right)-K \Phi\left(d_{-}(0)\right), \\
\Phi(x)=\int_{-\infty}^{x} \varphi(y) d y, \quad \varphi(y)=\frac{1}{\sqrt{2 \pi}} \exp \left\{-\frac{y^{2}}{2}\right\}, \\
d_{ \pm}(t)=\frac{\ln \left(S_{t} / K\right) \pm(T-t) \sigma^{2} / 2}{\sigma \sqrt{T-t}}, \quad t \in[0, T] .
\end{gathered}
$$

The formula for ${ }_{T} U_{x}$ given above has a very obvious meaning, which is derived from the structure of the payment in the contract under consideration.

The first component, ${ }_{T} p_{x} K$, represents the necessity of paying the quantity $K$ if the client lives (with probability ${ }_{T} p_{x}$ ) until the termination of the contract.

The second component shows the risk of mortality of the client with ${ }_{T} p_{x}$ and also the market risk arising from the presence of $\left(S_{T}-K\right)^{+}$. Quantitatively, such risk is valuated with the help of the price of a buyer's option, which consequently leads to the presence of the Black-Scholes formula in the structure of the premium ${ }_{T} p_{x}$.

Note that at the time when the contract is signed, the corresponding premium should equal

$$
{ }_{T-t} U_{x+t}={ }_{T-t} p_{x+t} K+{ }_{T-t} p_{x+t} C_{T}\left(t, S_{t}\right)
$$

where $C_{T}\left(t, S_{t}\right)$ is the capital of the minimal hedge for a buyer's option at time $t \in[0, T]$.

It is also known (see [7) that the price function, $C_{T}(t, x)$, satisfies the fundamental equation of Black and Scholes:

$$
\begin{gathered}
\frac{\partial C_{T}}{\partial t}+\frac{\sigma^{2}}{2} x^{2} \frac{\partial^{2} C_{T}}{\partial x^{2}}=0 . \\
\text { 2. Methodology of CALCulations in the } \\
\text { Black-SCholes Model With Stochastic VOlatility }
\end{gathered}
$$

The derivation of formulas (11)-(2) is based on the completeness of the Black-Scholes model. Probabilistically, completeness means that we have a unique risk-neutral, or martingale, measure $\hat{\mathrm{P}}^{*}$ and, consequently, a unique quantity $C_{T}$. These conclusions should remain true then in the case of a Black-Scholes market with a stochastic volatility, substituting $C_{0}$ and $C_{T}(t)$ for

$$
\sup _{\hat{\mathrm{P}}^{*}} \hat{\mathrm{E}}^{*}\left(S_{T}-K\right)^{+} \quad \text { and } \quad \underset{\hat{\mathrm{P}} *}{\operatorname{ess} \sup } \hat{\mathrm{E}}^{*}\left(\left(S_{T}-K\right) \mid \hat{F}_{t}\right),
$$

accordingly, where $\hat{\mathrm{P}}^{*}$ runs over all the different martingale measures in this incomplete market.

To realize this idea and find concrete values of the supremums above, we must modify the Black-Scholes formula to take into account stochastic volatility ([7]):

$$
d S_{t}=S_{t}\left(\mu d t+\sigma d W_{t}\right),
$$

where

$$
\sigma_{t}^{2}=\sigma^{2}+(-1)^{\Pi_{t}} \Delta \sigma^{2}
$$

and $\Pi_{t}$ is a Poisson process with unit intensity, $\Delta \sigma^{2} \ll \sigma^{2}$.

In this case, volatility is a random process that takes two values, $\sigma_{ \pm}=\sqrt{\sigma^{2} \pm \Delta \sigma^{2}}$.

Introducing a new process $\alpha=\left(\alpha_{t}\right)_{t \geq 0}$ with values $\sigma_{ \pm}$, we can view the price of the security $S_{t}$ as a controlled diffusion process $S_{t}^{\alpha}$. Taking this into account, the capital of the minimal hedge for $\left(S_{T}-K\right)^{+}$equals the price of the extremal problem

$$
\nu(t, x)=\sup _{\alpha} \hat{\mathrm{E}}\left(\left(S_{T}^{\alpha}-K\right)^{+} \mid S_{t}^{\alpha}=x\right)
$$


and, consequently, satisfies Bellman's differential equation (see [7, 8])

$$
\begin{gathered}
\frac{\partial \nu}{\partial t}+\frac{1}{2} \sigma^{2} x^{2} \frac{\partial^{2} \nu}{\partial x^{2}}+\frac{1}{2} \Delta \sigma^{2}\left|\frac{\partial^{2} \nu}{\partial x^{2}}\right|=0, \\
\nu(t, x)=(x-K)^{+}, \quad x \in \mathbf{R}_{+}, \quad t \in[0, T] .
\end{gathered}
$$

With $\Delta \sigma^{2}=0$, this equation becomes the Black-Scholes formula (3). Then we should build the solution of (4) from that of (3), with some corrections for the quantity $\Delta \sigma^{2} / \sigma^{2}$.

To realize this idea, let's use the change of variables for (4):

$$
\begin{gathered}
\xi=\ln x-\frac{\sigma^{2}}{2}(T-t), \\
\theta=\sigma^{2}(T-t) .
\end{gathered}
$$

Then for $\nu(t, x)=V(\theta, \xi)$ we get the following partial derivatives:

$$
\begin{gathered}
\frac{\partial \nu}{\partial t}=\sigma^{2}\left(\frac{1}{2} \frac{\partial V}{\partial \xi}-\frac{\partial V}{\partial \theta}\right), \\
\frac{\partial \nu}{\partial x}=\frac{1}{x} \frac{\partial V}{\partial \xi}, \\
\frac{\partial^{2} \nu}{\partial x^{2}}=\frac{1}{x^{2}}\left(\frac{\partial^{2} V}{\partial \xi^{2}}-\frac{\partial V}{\partial \xi}\right),
\end{gathered}
$$

and for the function $V(\theta, \xi)$ we get an equation equivalent to (4):

$$
\begin{gathered}
\frac{\partial V}{\partial \theta}=\frac{1}{2} \frac{\partial^{2} V}{\partial \xi^{2}}+\frac{1}{2} \frac{\Delta \sigma^{2}}{\sigma^{2}}\left|\frac{\partial^{2} V}{\partial \xi^{2}}-\frac{\partial V}{\partial \xi}\right|, \\
V(0, \xi)=\left(e^{\xi}-K\right)^{+} .
\end{gathered}
$$

We will not aim to find the solution to (5) directly, as this is rather difficult. Instead, we will find the approximate solution of

$$
V(\theta, \xi)=V_{0}(\theta, \xi)+\Delta \sigma^{2} V_{1}(\theta, \xi)+o\left(\Delta \sigma^{2}\right) .
$$

Substituting (6) into (5) and equating the terms of order zero and one with respect to $\Delta \sigma^{2}$, we get

$$
\begin{gathered}
\frac{\partial V_{0}}{\partial \theta}=\frac{1}{2} \frac{\partial^{2} V_{0}}{\partial \xi^{2}}, \quad V_{0}(0, \xi)=\left(e^{\xi}-K\right)^{+}, \\
\frac{\partial V_{1}}{\partial \theta}=\frac{1}{2} \frac{\partial^{2} V_{1}}{\partial \xi^{2}}+\frac{1}{2 \sigma^{2}}\left|\frac{\partial^{2} V_{0}}{\partial \xi^{2}}-\frac{\partial V_{0}}{\partial \xi}\right|, \quad V_{1}(0, \xi)=0 .
\end{gathered}
$$

Equation (7) is the same as that of Black-Scholes (3), and its solution has the form (see [9], for example):

$$
V_{0}(\theta, \xi)=\exp \left\{\xi+\frac{\theta}{2}\right\} \Phi\left(\frac{\xi+\theta-\ln K}{\sqrt{\theta}}\right)-K \Phi\left(\frac{\xi-\ln K}{\sqrt{\theta}}\right) .
$$

From (9) we get the following partial derivatives:

$$
\begin{aligned}
\frac{\partial V_{0}}{\partial \xi}= & \exp \left\{\xi+\frac{\theta}{2}\right\}\left(\Phi\left(\frac{\xi+\theta-\ln K}{\sqrt{\theta}}\right)+\frac{1}{\sqrt{\theta}} \varphi\left(\frac{\xi+\theta-\ln K}{\sqrt{\theta}}\right)\right)-\frac{K}{\sqrt{\theta}} \varphi\left(\frac{\xi-\ln K}{\sqrt{\theta}}\right), \\
\frac{\partial^{2} V_{0}}{\partial \xi^{2}}= & \exp \left\{\xi+\frac{\theta}{2}\right\}\left(\Phi\left(\frac{\xi+\theta-\ln K}{\sqrt{\theta}}\right)+\left(1+\frac{2}{\sqrt{\theta}}-\frac{\xi-\ln K}{\theta}\right) \varphi\left(\frac{\xi+\theta-\ln K}{\sqrt{\theta}}\right)\right) \\
& +\frac{K}{\sqrt{\theta}}\left(\frac{\xi-\ln K}{\sqrt{\theta}}\right) \varphi\left(\frac{\xi-\ln K}{\sqrt{\theta}}\right) .
\end{aligned}
$$


Then, taking into account the following equality:

$$
\varphi\left(\frac{\xi+\theta-\ln K}{\sqrt{\theta}}\right)=K \exp \left\{-\left(\xi+\frac{\theta}{2}\right)\right\} \varphi\left(\frac{\xi-\ln K}{\sqrt{\theta}}\right),
$$

we have, for the nonlinear term in (8),

$$
\frac{\partial^{2} V_{0}}{\partial \xi^{2}}-\frac{\partial V_{0}}{\partial \xi}=\frac{K}{\sqrt{\theta}} \varphi\left(\frac{\xi-\ln K}{\sqrt{\theta}}\right) \geq 0
$$

thus

$$
V_{1}(\theta, \xi)=\frac{K \sqrt{\theta}}{2 \sigma^{2}} \varphi\left(\frac{\xi-\ln K}{\sqrt{\theta}}\right) .
$$

As a result, we find that

$$
\begin{aligned}
\nu\left(S_{0}, 0\right) \cong & S_{0} \Phi\left(\frac{\ln \left(S_{0} / K\right)+\sigma^{2} T / 2}{\sigma \sqrt{T}}\right)-K \Phi\left(\frac{\ln \left(S_{0} / K\right)-\sigma^{2} T / 2}{\sigma \sqrt{T}}\right) \\
& +\frac{K \Delta \sigma^{2}}{2 \sigma^{2}} \sigma \sqrt{T} \varphi\left(\frac{\ln \left(S_{0} / K\right)-\sigma^{2} T / 2}{\sigma \sqrt{T}}\right) .
\end{aligned}
$$

In view of (9), we derive the following formula for a premium in the model of BlackScholes with stochastic volatility:

$$
{ }_{T} U_{x}={ }_{T} p_{x} K+{ }_{T} p_{x} C_{T}(0)+{ }_{T} p_{x} \frac{\Delta \sigma^{2}}{2 \sigma^{2}} K \sigma \sqrt{T} \varphi\left(\frac{\ln \left(S_{0} / K\right)-\sigma^{2} T / 2}{\sigma \sqrt{T}}\right) .
$$

\section{Concluding Remarks}

Formulas for ${ }_{T} U_{x}$ presented above utilize the methods of perfect hedging and superhedging. Note that in the works [5, 6] mean-variance hedging was used for the BlackScholes model. We can extend this kind of hedging to a more general Gaussian model of the type

$$
S_{t}=\exp \left\{Y_{t}-\frac{1}{2}\left\langle Y_{t}\right\rangle\right\}
$$

where $Y$ is a Gaussian martingale with quadratic characteristic $\langle Y\rangle_{t}$ (see [10]).

To the explanations given for our methods of valuation of the flexible schemes of life insurance, we can add the following ideas.

As a basis of a pure endowment contract with guarantee, for example, we can take this payment obligation:

$$
\max \left\{S_{T}, K\right\} \cdot I_{T(x)>T},
$$

which is dominated by the obligation

$$
\max \left\{S_{T}, K\right\} .
$$

In the standard Black-Scholes model (see part 1) this purely market obligation allows a perfect hedge with an initial price

$$
K+C_{T}(0)
$$

Formula (1) for ${ }_{T} U_{x}$ represents

$$
c\left(K+C_{T}(0)\right) \leq K+C_{T}(0),
$$

where

$$
0 \leq c={ }_{T} p_{x} \leq 1
$$

Inequality (13) can be viewed as a budget constraint on the exercise of the option with payment (12). For such options, generally speaking, strategies for perfect hedging, which accumulate enough capital to cover the payment no matter what direction the market 
takes, do not exist. However, with the help of the quantile hedging method, we can build a hedge that maximizes the probability of perfect hedging ([7]). This hedge should be used for a mixed obligation (11), with the quantity

$$
c\left(K+C_{T}(0)\right)
$$

received as a premium ${ }_{T} U_{x}$. In this situation, valuation of premiums ${ }_{T} U_{x}$ suggested here allows the insurance company to minimize the risk of not being able to make payments on such contracts.

\section{BIBLIOGRAPHY}

1. N. L. Bowers, H. U. Gerber, J. C. Hickman, D. A. Jones, and C. I. Nesbitt, Actuarial Mathematics, Society of Actuaries, Schaumburg, Illinois, 1997.

2. M. J. Brennan and E. S. Schwartz, The pricing of equity-linked life insurance policies with an asset value guarantee, Journal of Financial Economics 3 (1976), 195-213.

3. K. Aase and S. Persson, Pricing of unit-linked insurance policies, Scandinavian Actuarial Journal 1 (1994), 26-52.

4. P. P. Boyle and M. R. Hardy, Reserving for maturity guarantees: Two approaches, Insurance: Mathematics and Economics 21 (1997), 113-127.

5. T. Møller, Risk-minimizing hedging strategies for unit-linked life-insurance contracts, Astin Bulletin 28 (1998), 17-47.

6. T. Møller, Hedging equity-linked life insurance contracts, North American Actuarial Journal 5 (2001), no. 2, 79-95. MR2012038

7. A. V. Melnikov, S. N. Volkov, and M. L. Nechaev, Mathematics of Financial Obligations, AMS, Providence, RI, 2002. MR:1918716 (2003f:91055)

8. N. V. Krylov, Controlled Diffusion Processes, Springer, 1980. MR601776 (82a:60062)

9. V. S. Vladimirov, Equations of Mathematical Physics, "Nauka", Moscow, 1982. (Russian) MR0653331 (83i:00029)

10. A. V. Melnikov and M. L. Nechaev, On the calculations of flexible life insurance contracts in Gaussian financial environment (2003) (to appear).

Steklov Mathematical Institute, Russian Academy of Sciences, Moscow, Russia

Current address: Department of Mathematical and Statistical Sciences, University of Alberta, Edmonton, Canada

E-mail address: melnikov@ualberta.ca

Efficient Capital Management, LlC, Naperville, Illinois

E-mail address: marat@efficientcapital.com

Mercer Investment Consulting, Montreal, Canada

E-mail address: vskorn@shaw.ca

Received 17/MAR/2003

Originally published in English 